Advanced Courses in Mathematics CRM Barcelona

Centre de Recerca Matemàtica

Managing Editor:

Manuel Castellet 
Silvia Bertoluzza

Silvia Falletta

Giovanni Russo

Chi-Wang Shu

\section{Numerical Solutions of Partial Differential Equations}

Basel · Boston · Berlin 
Authors:

Silvia Bertoluzza

Istituto di Matematica Applicata e

Tecnologie Informatiche del C.N.R.

v. Ferrata 1

27100 Pavia

Italy

e-mail: silvia.bertoluzza@imati.cnr.it

Silvia Falletta

Dipartimento di Matematica

Politecnico di Torino

Corso Duca degli Abruzzi, 24

10129 Torino

Italy

e-mail: silvia.falletta@polito.it
Giovanni Russo

Dipartimento di Matematica ed Informatica

Università di Catania

Viale Andrea Doria 6

95125 Catania

Italy

e-mail: russo@dmi.unict.it

Chi-Wang Shu

Division of Applied Mathematics

Brown University

Providence, RI 02912

USA

e-mail:shu@dam.brown.edu

2000 Mathematical Subject Classification 35-99, 65-99

Library of Congress Control Number: 2008940758

Bibliographic information published by Die Deutsche Bibliothek

Die Deutsche Bibliothek lists this publication in the Deutsche Nationalbibliografie;

detailed bibliographic data is available in the Internet at 〈http://dnb.ddb.de $\rangle$.

\section{ISBN 978-3-7643-8939-0 Birkhäuser Verlag AG, Basel - Boston - Berlin}

This work is subject to copyright. All rights are reserved, whether the whole or part of the material is concerned, specifically the rights of translation, reprinting, re-use of illustrations, recitation, broadcasting, reproduction on microfilms or in other ways, and storage in data banks. For any kind of use permission of the copyright owner must be obtained.

(c) 2009 Birkhäuser Verlag, P.O. Box 133, CH-4010 Basel, Switzerland

Part of Springer Science+Business Media

Printed on acid-free paper produced from chlorine-free pulp. TCF $\infty$

Printed in Germany

ISBN 978-3-7643-8939-0

987654321
e-ISBN 978-3-7643-8940-6

www.birkhauser.ch 


\section{Contents}

Foreword $\quad$ ix

I Wavelets and Partial Differential Equations

Silvia Bertoluzza and Silvia Falletta 1

Introduction . . . . . . . . . . . . . . . . . . . 3

1 What is a Wavelet? 5

1.1 Multiresolution Analysis . . . . . . . . . . . . . . . 5

1.1.1 Example I: The Haar Basis . . . . . . . . . . . . . . . . 13

1.1 .2 Example II: B-Splines . . . . . . . . . . . . . . . . . 14

1.1.3 Example III: Daubechies's Wavelets . . . . . . . . . . . . . 14

1.1.4 Example IV: The Schauder Basis . . . . . . . . . . . . 15

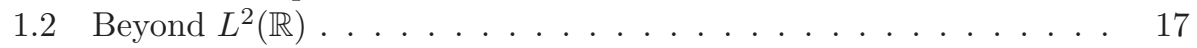

2 The Fundamental Property of Wavelets 23

2.1 The case $\Omega=\mathbb{R}$ : The Frequency Domain Point of View vs.

The Space Domain Point of View . . . . . . . . . . . . . . . . 24

2.2 The General Case: $\Omega$ Domain of $\mathbb{R}^{d} \ldots$. . . . . . . . . . . . . . . 32

2.3 The Issue of Boundary Conditions . . . . . . . . . . . . . . . 35

3 Wavelets for Partial Differential Equations $\quad 37$

3.1 Wavelet Preconditioning . . . . . . . . . . . . . . . . . . 37

3.2 Nonlinear Wavelet Methods for the Solution of PDEs . . . . . . . . 41

3.2.1 Nonlinear vs. Linear Wavelet Approximation . . . . . . . . 41

3.2.2 Nonlinear Solution of PDEs . . . . . . . . . . . . . . . . . 44

3.3 Wavelet Stabilisation of Unstable Problems . . . . . . . . . . . . . 47

3.4 A-Posteriori Error Estimates . . . . . . . . . . . . . . . . . 50

3.5 Operations on Infinite Matrices and Vectors . . . . . . . . . . . . 51

$\begin{array}{ll}\text { Bibliography } & 55\end{array}$ 


\section{High Order Shock-Capturing Schemes for Balance Laws}

Giovanni Russo

$\begin{array}{lll}1 & \text { Introduction } & 61\end{array}$

1.1 Hyperbolic Systems . . . . . . . . . . . . . . . . . . . . 62

1.2 Simple Three-Point Schemes . . . . . . . . . . . . . . . . . . 64

1.3 Conservation Form, Jump Conditions and Conservative Schemes . 68

1.4 Consistency and Convergence . . . . . . . . . . . . . . . 72

1.5 Conservation Properties . . . . . . . . . . . . . . . 72

1.6 Entropy Condition . . . . . . . . . . . . . . . . . . . . 73

1.7 Discrete Entropy Condition . . . . . . . . . . . . . . . . 75

1.8 Dissipation, Dispersion, and the Modified Equation . . . . . . . . . 75

1.9 Second-Order Methods and Dispersion . . . . . . . . . . . . . 77

$2 \quad$ Upwind Scheme for Systems $\quad 83$

2.1 The Riemann Problem . . . . . . . . . . . . . . . . . 85

2.2 Godunov Scheme . . . . . . . . . . . . . . . 85

3 The Numerical Flux Function $\quad \mathbf{8 9}$

3.1 Higher-Order Extensions of the Godunov Method . . . . . . . . . . 89

3.2 The Scalar Equation and Monotone Fluxes . . . . . . . . . . . . . 90

4 Nonlinear Reconstruction and High-Order Schemes $\quad 97$

4.1 High-Order Finite-Volume Schemes . . . . . . . . . . . . . . . . . . 97

4.2 Essentially Non-Oscillatory Reconstruction (ENO) . . . . . . . . . 99

4.3 Weighted ENO Reconstruction (WENO) . . . . . . . . . . . . . 102

4.4 Conservative Finite-Difference Schemes . . . . . . . . . . . . . . . . 104

4.5 Time Integration: Runge-Kutta Methods . . . . . . . . . . . . . . 106

4.6 SSP Schemes . . . . . . . . . . . . . . . . . 107

4.7 Extension to More Dimensions . . . . . . . . . . . . . . 108

5 Central Schemes 109

5.1 Nessyahu-Tadmor Second-Order Scheme . . . . . . . . . . . . . . . 110

5.2 Description of CRK Schemes . . . . . . . . . . . . . . . . . 112

5.3 A Second-Order Scheme: CRK2 . . . . . . . . . . . . . . . 114

5.4 Higher-Order Schemes: CRK3, CRK4, CRK5 . . . . . . . . . . . . 114

5.5 Numerical Tests . . . . . . . . . . . . . . . . . . . . . 118

5.6 Systems of Equations . . . . . . . . . . . . . . . . . . . . . 119

5.7 Componentwise Application . . . . . . . . . . . . . . . . . . . 120

5.8 Projection Along Characteristic Directions . . . . . . . . . . . . 123 
6 Systems with Stiff Source $\quad \mathbf{1 2 5}$

6.1 Systems of Balance Laws . . . . . . . . . . . . . . . . . . . 126

6.2 IMEX Runge-Kutta Schemes . . . . . . . . . . . . . . . . . . . . 128

6.3 Hyperbolic Systems with Relaxation . . . . . . . . . . . . . 130

6.3.1 Zero Relaxation Limit . . . . . . . . . . . . . . . . . . 130

6.3.2 Asymptotic Properties of IMEX Schemes . . . . . . . . . . 132

6.4 Numerical Tests . . . . . . . . . . . . . . . . . . . . . . . . . . . 134

6.4.1 Broadwell Model . . . . . . . . . . . . . . . . . . 134

6.4 .2 Shallow Water . . . . . . . . . . . . . . . . 135

6.4.3 Traffic Flows . . . . . . . . . . . . . . 137

Appendix: Butcher Tableau of IMEX-RK 141

$\begin{array}{ll}\text { Bibliography } & 143\end{array}$

\section{Discontinuous Galerkin Methods:} General Approach and Stability Chi-Wang Shu

Preface

1 Introduction $\quad 153$

2 Time Discretization $\quad 155$

3 Discontinuous Galerkin Method for Conservation Laws $\quad 157$

3.1 Two-dimensional Steady-State Linear Equations . . . . . . . . . . 157

3.2 One-dimensional Time-dependent Conservation Laws . . . . . . . . 160

3.2.1 Cell Entropy Inequality and $L^{2}$-Stability . . . . . . . . . . . 162

3.2.2 Limiters and Total Variation Stability . . . . . . . . . . . . 164

3.2.3 Error Estimates for Smooth Solutions . . . . . . . . . . 168

3.3 Comments for Multi-dimensional Cases . . . . . . . . . . . . . 170

4 Discontinuous Galerkin Method for Convection-Diffusion Equations $\quad \mathbf{1 7 5}$

4.1 LDG Scheme Formulation . . . . . . . . . . . . . . . . 176

4.2 Stability Analysis . . . . . . . . . . . . . . . . . . . 177

4.3 Error Estimates . . . . . . . . . . . . . . . . . . . . . 179

4.4 Multi-Dimensions . . . . . . . . . . . . . . . . . . . . . 181

5 Discontinuous Galerkin Method for PDEs Containing

Higher-Order Spatial Derivatives $\quad 183$

5.1 LDG Scheme for the KdV Equations . . . . . . . . . . . . . . . . . . . 183

5.1 .1 Stability Analysis . . . . . . . . . . . . . . . . . 185

5.1 .2 Error Estimates . . . . . . . . . . . . . . 187 
5.2 LDG Schemes for Other Higher-Order PDEs . . . . . . . . . . . 190

5.2.1 Bi-harmonic Equations . . . . . . . . . . . . . 190

5.2.2 Fifth-Order Convection-Dispersion Equations . . . . . . . . 191

5.2 .3 The $K(m, n)$ Equations . . . . . . . . . . . . . . . . . . 191

5.2.4 The KdV-Burgers-Type (KdVB) Equations . . . . . . . . . 191

5.2.5 The Fifth-Order KdV-Type Equations . . . . . . . . . . . . 192

5.2.6 The Fully Nonlinear $K(n, n, n)$ Equations . . . . . . . . . . 192

5.2.7 The Nonlinear Schrödinger (NLS) Equation . . . . . . . . . 192

5.2.8 The Kadomtsev-Petviashvili (KP) Equations . . . . . . . . 193

5.2.9 The Zakharov-Kuznetsov (ZK) Equation . . . . . . . . . . . 193

5.2.10 The Kuramoto-Sivashinsky-type Equations . . . . . . . . . 193

5.2.11 The Ito-Type Coupled KdV Equations . . . . . . . . . . . . 194

5.2.12 The Camassa-Holm (CH) Equation . . . . . . . . . . . . . . 194

5.2.13 The Cahn-Hilliard Equation . . . . . . . . . . . . . . . 194 


\section{Foreword}

This book contains an expanded and smoothed version of lecture notes delivered by the authors at the Advanced School on Numerical Solutions of Partial Differential Equations: New Trends and Applications, which took place at the Centre de Recerca Matemàtica (CRM) in Bellaterra (Barcelona) from November 15th to 22nd, 2007.

The book has three parts. The first part, by Silvia Bertoluzza and Silvia Falletta, is devoted to the use of wavelets to derive some new approaches in the numerical solution of PDEs, showing in particular how the possibility of writing equivalent norms for the scale of Besov spaces allows to write down some new methods. The second part, by Giovanni Russo, provides an overview of the modern finite-volume and finite-difference shock-capturing schemes for systems of conservation and balance laws, with emphasis in giving a unified view of such schemes by identifying the essential aspects of their construction. In the last part Chi-Wang Shu gives a general introduction to the discontinuous Galerkin methods for solving some classes of PDEs, discussing cell entropy inequalities, nonlinear stability and error estimates.

The school that originated these notes was born with the objective of providing an opportunity for $\mathrm{PhD}$ students, recent $\mathrm{PhD}$ doctorates and researchers in general in fields of applied mathematics and engineering to catch up with important developments in the fields and/or to get in touch with state-of-the-art numerical techniques that are not covered in usual courses at graduate level.

We are indebted to the Centre de Recerca Matemàtica and its staff for hosting the advanced school and express our gratitude to José A. Carrillo (Institució Catalana de Recerca i Estudis Avançats - Universitat Autònoma de Barcelona), Rosa Donat (Universitat de Valéncia), Carlos Parés (Universidad de Málaga) and Yolanda Vidal (Universitat Politècnica de Catalunya) for the mathematical organisation of the course and for making it such a pleasant experience. 